\title{
Human Immunodeficiency Virus Arrests Plasmacytoid Dendritic Cells in a Granzyme B ${ }^{\text {high }}$ Tolerogenic State
}

\author{
Bernd Jahrsdörfer ${ }^{1 \#}$, Verena Panitz ${ }^{2}$ and Dorit Fabricius ${ }^{2 * \#}$ \\ ${ }^{1}$ Laboratory of Cellular Immunology, Institute of Clinical Transfusion Medicine and Immunogenetics, Associate Professor of Clinical Pharmacology, Ulm University, \\ Helmholtzstrasse 10, Ulm-8908, Germany \\ ${ }^{2}$ Department of Pediatrics, Ulm University, Eythstrasse 24, Ulm-89075, Germany
}

\#Authors are equally contributed

"Corresponding author: Dorit Fabricius, Assistant Professor of Pediatrics, Department of Pediatrics, Ulm University Eythstrasse, 2489075 Ulm, Germany, Tel: +49 731 500 57376; Fax: +49 731500 57042; E-mail: dorit.fabricius@uni-ulm.de

Received date: Jul 25, 2016; Accepted date: Aug 25, 2016; Published date: Aug 29, 2016

Copyright: (c) 2016 Jahrsdörfer B, et al. This is an open-access article distributed under the terms of the Creative Commons Attribution License, which permits unrestricted use, distribution, and reproduction in any medium, provided the original author and source are credited.

\section{Short Communication}

The serine protease granzyme B (GzmB) is classically known to be an apoptogenic effector molecule produced by cytotoxic cells including NK cells and CD8+ T lymphocytes [1]. However, a series of further cell types are able to express GzmB, most of them in the absence of perforin. Below them are different antigen-presenting cells (APC) including $\mathrm{B}$ cells, monocyte-derived dendritic cells and plasmacytoid dendritic cells (pDC) [2-7]. Here, we report that GzmB contributes to a variety of dendritic cell-related functions including antigen uptake and $\mathrm{T}$ cell regulation, and that HIV can impact on these functions.

Recent years have uncovered a variety of non-cytotoxic roles of GzmB $[8,9]$. Of note, the cytotoxic function of T cells and NK cells results from GzmB being secreted in the presence of the pore-forming protein perforin (Pfn). This allows GzmB to reach the cytosol of target cells after exocytosis, where it may then activate apoptosis-inducing enzymes including caspases, DNAses and BID [1]. In contrast, certain immune cells including plasmacytoid dendritic cells (pDC) and B cells can produce GzmB only, but not Pfn [6,10-12]. GzmB secreted by these APC types therefore primarily encounters substrates with an extracellular or membrane-close localisation such as extracellular proteins or membrane-bound receptors [8]. As a result, these cells develop a strong immunoregulatory potential based on GzmBdependent cleavage of the $\mathrm{T}$ cell receptor (TCR)- $\zeta$-chain [6,9-13]. Degradation of the TCR- $\zeta$-chain by GzmB directly limits the proliferative capacity and survival of effector $\mathrm{T}$ cells.

GzmB produced by APC however may not only play an important role for their interaction with $\mathrm{T}$ cells, but also for antigen processing. Recently, GzmB was shown to enhance both the uptake of antigens by release of "eat-me" signals on dying cells as well as their crosspresentation $[14,15]$. Importantly, the cleavage of peptides by GzmB can result in fragments that represent neo-antigens, allowing the establishment of increased immune responses towards such antigens $[16,17]$. This suggests that antigens generated by GzmB are principally more immunogenic than antigens generated by other proteases. If GzmB-borne antigens arise, for example in the course of a $\mathrm{T}$ cellmediated attack of virus-infected cells, they may pose a substantial risk of triggering autoimmune responses. Therefore, GzmB produced by pDC and other APC is partly secreted into the extracellular space, where it can prevent early activation of unspecific $\mathrm{T}$ cells as described above (Figure 1, middle panel).

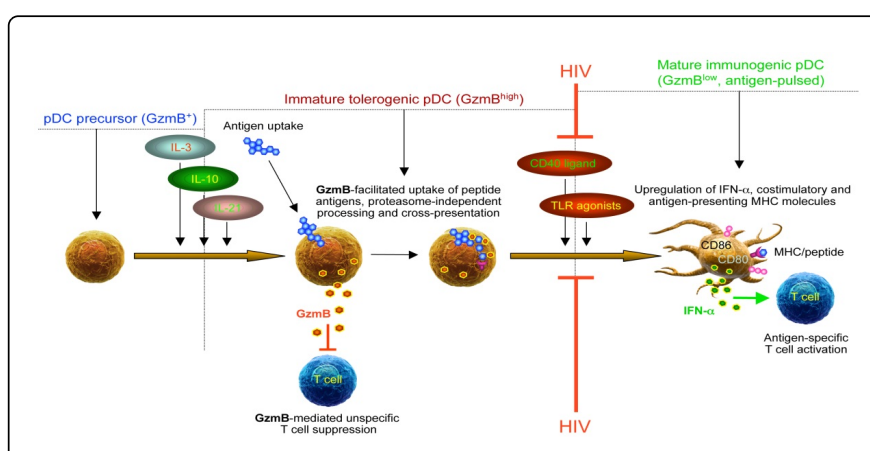

Figure 1: Current hypothesis for the maturation of plasmacytoid dendritic cells. Maturation of pDC precursors occurs in a biphasic process. During the first phase, pDC develop a tolerogenic phenotype in response to different cytokines including IL-3, IL-10 and IL-21. This phenotype involves the production of large amounts of the serine protease GzmB, which is involved not only in the suppression of effector $\mathrm{T}$ cell responses, but also in antigen uptake and proteasome-independent antigen processing. In the second phase, pDCs are rapidly (within 24 hours) matured in response to TLR ligands and CD40 ligand. GzmB is downmodulated, while costimulatory molecules and $\mathrm{MHC} /$ peptide complexes are simultaneously upregulated, resulting in a highly immunogenic and mature pDC phenotype. Our data suggest that the transition of pDC into this second phase is blocked during acute HIV infection, possibly by virus-associated factors antagonizing CD40- and TLRmediated signals.

Simultaneously, GzmB facilitates antigen uptake and processing into immunogenic fragments for cross-presentation. The GzmB high phase of pDC maturation is subsequently followed by a second phase, initiated by toll-like-receptor (TLR) ligand- and CD40 ligand-induced signals. These signals downregulate GzmB and induce co-stimulatory as well as antigen-presenting molecules on the pDC surface (Figure 1, right panel). Current data from our laboratory demonstrate that HIV enhances and maintains an immature and GzmB high phenotype in peripheral blood-derived pDC (Figure 2). This suggests that the transition of pDC into the second maturation phase is blocked by HIV, resulting in the persistence of a $\mathrm{GzmB}^{\text {high }}$ tolerogenic state during HIV infection (Figure 1). Of note, while expression of GzmB is part of the maturity state of pDC, it is not believed to be causally involved in the transition of $\mathrm{pDC}$ from an immature into a mature state. Instead, virus-associated factors antagonizing CD40- or TLR-mediated signals 
may be responsible for the observed transition arrest $[6,10]$. Support for our hypothesis comes from an independent group, which demonstrated that gut-associated pDC from patients with HIV infection are also characterized by an immature phenotype and enhanced GzmB expression [18].

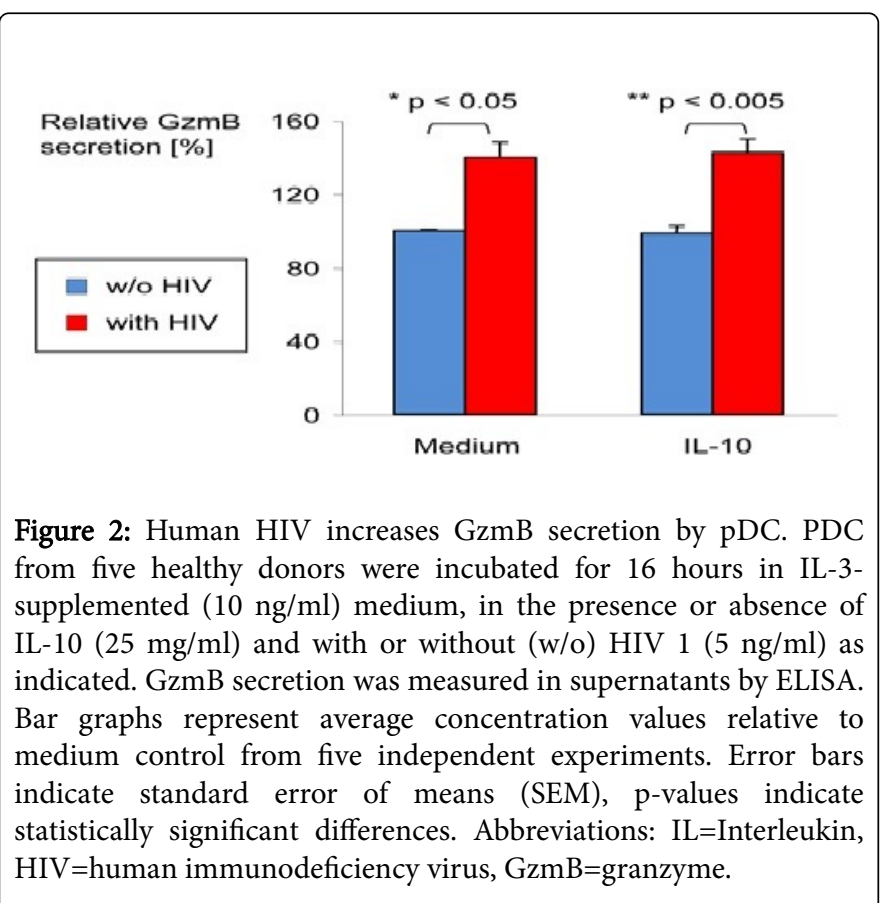

Overall, our data suggest the maturation of $\mathrm{pDC}$ involves a dynamic process of GzmB expression and secretion, which is connected to different $\mathrm{pDC}$ functions and which is influenced by HIV. Further studies are necessary to elucidate, whether GzmB produced by $\mathrm{pDC}$ may serve as a target for therapeutic manipulation of immune responses after viral infections such as with HIV and during pDCbased vaccinations. For example, the suppression of $\mathrm{pDC}$ by pharmacological means, or direct neutralization of GzmB secreted by pDC may allow a better establishment of cellular antiviral immune responses during infection, resulting in faster clearance of virusinfected cells.

\section{References}

1. Lord SJ, Rajotte RV, Korbutt GS, Bleackley RC (2003) Granzyme B: a natural born killer. Immunol Rev 193: 31-38.

2. Jahrsdörfer B, Blackwell SE, Wooldridge JE, Huang J, Andreski MW, et al. (2006) B chronic lymphocytic leukemia cells and other B cells can produce granzyme B and gain cytotoxic potential after interleukin-21based activation. Blood 108: 2712-2719.
3. Hagn M, Schwesinger E, Ebel V, Sontheimer K, Maier J, et al. (2009) Human B cells secrete granzyme B when recognizing viral antigens in the context of the acute phase cytokine IL-21. J Immunol 183: 1838-1845.

4. Schuldhaus C, Beyer T, Kaltenmeier C, Lindner S, Schrezenmeier H, et al. (2013) Monocytes express the serine protease granzyme B - potential involvement in antigen processing and presentation. J Immunol 190 Supplemental.

5. Korthals M, Safaian N, Kronenwett R, Maihofer D, Schott M, et al. (2007) Monocyte derived dendritic cells generated by IFN-alpha acquire mature dendritic and natural killer cell properties as shown by gene expression analysis. J Transl Med 5: 46.

6. Jahrsdörfer B, Vollmer A, Blackwell SE, Maier J, Sontheimer J, et al. (2010) Granzyme B produced by human plasmacytoid dendritic cells suppresses T-cell expansion. Blood 115: 1156-1165.

7. Rissoan MC, Duhen T, Bridon JM, Bendriss-Vermare N, Peronne C, et al. (2002) Subtractive hybridization reveals the expression of immunoglobulin-like transcript 7, Eph-B1, granzyme B, and 3 novel transcripts in human plasmacytoid dendritic cells. Blood 100: 3295-3303.

8. Buzza MS, Bird PI (2006) Extracellular granzymes: current perspectives. Biol Chem 387: 827-837.

9. Hagn M, Jahrsdörfer B (2012) Why do human B cells secrete granzyme $\mathrm{B}$ ? Insights into a novel B-cell differentiation pathway. Oncoimmunology 1: $1368-1375$.

10. Fabricius D, Nussbaum B, Busch D, Panitz V, Mandel B, et al. (2013) Antiviral vaccines license $\mathrm{T}$ cell responses by suppressing granzyme $\mathrm{B}$ levels in human plasmacytoid dendritic cells. J Immunol 191: 1144-1153.

11. Lindner S, Dahlke K, Sontheimer K, Hagn M, Kaltenmeier C, et al. (2013) Interleukin 21-Induced Granzyme B-Expressing B Cells Infiltrate Tumors and Regulate T Cells. Cancer Res 73: 2468-2479.

12. Kaltenmeier C, Gawanbacht A, Beyer T, Lindner S,Trzaska T, et al. (2015) CD4+ T cell-derived IL-21 and deprivation of CD40 signaling favor the in vivo development of granzyme B-expressing regulatory B cells in HIV patients. jimmunol 194: 3768-3777.

13. Wieckowski E, Wang GQ, Gastman BR, Goldstein LA, Rabinowich H (2002) Granzyme B-mediated degradation of T-cell receptor zeta chain. Cancer Res 62: 4884-4889.

14. Hoves S, Sutton VR, Haynes NM, Hawkins ED, Ruiz FD, et al. (2011) A critical role for granzymes in antigen cross-presentation through regulating phagocytosis of killed tumor cells. J Immunol 187: 1166-1175.

15. Hoves S, Sutton VR, Trapani JA (2012) A novel role for granzymes in anti-tumor immunity. Oncoimmunology 1: 219-221.

16. Casciola-Rosen L, Andrade F, Ulanet D, Wong WB, Rosen A. (1999) Cleavage by granzyme $\mathrm{B}$ is strongly predictive of autoantigen status: implications for initiation of autoimmunity. J Exp Med 190: 815-826.

17. Casciola-Rosen L, Miagkov A, Nagaraju K, Askin F, Jacobson L, et al. (2008) Granzyme B: evidence for a role in the origin of myasthenia gravis. J Neuroimmunol 201-202: 33-40.

18. Boichuk SV, Khaiboullina SF, Ramazanov BR, Khasanova GR, Ivanovskaya KA, et al. (2015) Gut-Associated Plasmacytoid Dendritic Cells Display an Immature Phenotype and Upregulated Granzyme B in Subjects with HIV/AIDS. Front Immunol 6: 485. 